\title{
Band alignment calculation of dielectric films on $\mathrm{VO}_{2}$
}

\author{
Zhaofu Zhang $^{1}$, Jiaqi Chen ${ }^{1}$, Yuzheng Guo ${ }^{2}$, and John Robertson ${ }^{1, \text { a) }}$ \\ ${ }^{1}$ Department of Engineering, Cambridge University, Cambridge, CB2 1PZ, United Kingdom \\ ${ }^{2}$ College of Engineering, Swansea University, Swansea, SA1 8EN, United Kingdom
}

a) E-mail address of corresponding author: jr214@cam.ac.uk

The chemical bonding and band edge line-up of several gate insulators on monoclinic (M1) phase $\mathrm{VO}_{2}$ are studied based on hybrid density functional calculations. High dielectric constant $\mathrm{HfO}_{2}$ and $\mathrm{ZrO}_{2}$, wide band gap oxide $\mathrm{Al}_{2} \mathrm{O}_{3}$, and narrower band gap $\mathrm{TiO}_{2}$ are considered. The insulating interface supercells with a clean bandgap are built and adapted to analysis the band alignment. All the gate insulators show the type-I band alignment with $\mathrm{VO}_{2}$. The valence band offset are all larger than $1 \mathrm{eV}$. The calculated conduction band offsets for $\mathrm{HfO}_{2}, \mathrm{ZrO}_{2}$ and $\mathrm{Al}_{2} \mathrm{O}_{3}$ are all larger than $2 \mathrm{eV}$. In terms of the band alignment, these three insulators can work as suitable gate dielectrics for $\mathrm{VO}_{2}$-based device application, but more accurate line-up analysis on $\mathrm{TiO}_{2} / \mathrm{VO}_{2}$ interface is still needed.

Keywords: vanadium dioxide, band alignment; metal-insulator transition FET, density functional calculation

\section{Introduction}

Vanadium dioxide $\left(\mathrm{VO}_{2}\right)$ undergoes a metal-insulator transition (MIT) from insulating monoclinic (M1) phase at lower temperatures to a metallic rutile phase above $\sim 340 \mathrm{~K}$ [1-3]. This makes $\mathrm{VO}_{2}$ useful as a thermochromic material in smart windows [4], RF switches [5], and steep slope transistors [6]. The latter application uses a field effect transistor (FET) device [7-9], with a gate oxide, but features different mechanisms than metal-oxide-semiconductor FET [10,11]. For efficient operation of this metal-insulator transition transistor, it is necessary for the band offsets (BOs) be sufficiently large (ideally, $>1 \mathrm{eV}$ ), like that in the MOSFET devices [12].

Multiple dielectric/ $\mathrm{VO}_{2}$ interfaces have been studied experimentally. Dielectric films such as $\mathrm{Al}_{2} \mathrm{O}_{3}[13,14]$ and $\mathrm{TiO}_{2}[15]$ have been deposited on $\mathrm{VO}_{2}$ to study the phase transition stability and electronic characteristics. Nemanich et al. utilized $\mathrm{VO}_{2}$ as an interlayer in a $\mathrm{HfO}_{2}-\mathrm{Si}$ gate stack and a $\mathrm{HfO}_{2}-\mathrm{VO}_{2}-\mathrm{HfO}_{2}$ confined well structure to study the band alignment $[16,17]$. Besides, highquality $\mathrm{VO}_{2}$ films can be epitaxially growth on $\mathrm{TiO}_{2}$ substrate by pulsed laser deposition, and tune the MIT transition by interfacial strain engineering [18]. Theoretical work on $\mathrm{VO}_{2}$ mostly focuses on the phase transition mechanism and band theory $[19,20]$. Thus, a detailed investigation of the dielectric $/ \mathrm{VO}_{2}$ interface properties, especially the band edge line-up based on the reasonable interface configurations is highly desirable.

In this work, we have therefore calculated the band offsets of the insulating $\mathrm{M} 1$ phase $\mathrm{VO}_{2}$ with various dielectric oxides, such as high dielectric constant $\mathrm{HfO}_{2}$ and $\mathrm{ZrO}_{2}$, wide band gap oxide $\mathrm{Al}_{2} \mathrm{O}_{3}$, and narrower band gap $\mathrm{TiO}_{2}$, using realistic atomic models of the interface supercells. Both the atomic structures and electronic properties are systematically studied. To derive the band line-up with $\mathrm{VO}_{2}$, we use both the partial density of states (PDOS) and the core-level scheme. Our results prove that the conduction band offset (CBO) values for $\mathrm{HfO}_{2}, \mathrm{ZrO}_{2}, \mathrm{Al}_{2} \mathrm{O}_{3}$ interface are all larger than $2 \mathrm{eV}$, and their valence band offsets $(\mathrm{VBO})$ are $\sim 1 \mathrm{eV}$. The band offset of $\mathrm{TiO}_{2}$ interface is somewhat lower, suffering from the severe underestimation of band gap on the $\mathrm{TiO}_{2}$ side.

\section{Methods}


The calculations in this work were conducted based on density functional theory (DFT), as implemented in the plane-wave CASTEP code [21]. The core-valence interaction was described by norm-conserving pseudopotential with a plane-wave cutoff energy of $680 \mathrm{eV}$. Geometry relaxations were conducted using the GGA-PBE exchange-correlation functional [22], with the force acting on each atom converged to $0.02 \mathrm{eV} / \AA$. Considering the bandgap underestimation in GGA, the electronic structures were calculated by Heyd-Scuseria-Ernzerhof (HSE) hybrid functional [23] with a Hartree-Fock (HF) exchange factor of $15 \%$ rather than the default value of $25 \%$, yielding a bandgap of $0.7 \mathrm{eV}$ for monoclinic (M1) phase $\mathrm{VO}_{2}$. Besides, Pantelides et al. reported that both energy gaps and magnetic ordering of monoclinic $\mathrm{VO}_{2}$ are consistent with the available experimental data with a smaller HF fraction [24]. However, using this HF fraction, the calculated bandgap of the gate dielectrics is inevitable underestimated referring to the experimental data. Calculated band gaps are $5.2 \mathrm{eV}, 4.7 \mathrm{eV}, 4.1 \mathrm{eV}$ and $2.3 \mathrm{eV}$ for $\mathrm{Al}_{2} \mathrm{O}_{3}, \mathrm{HfO}_{2}, \mathrm{ZrO}_{2}$, and $\mathrm{TiO}_{2}$, respectively, compared with the experimental counterpart of $6.5 \mathrm{eV}, 6.0 \mathrm{eV}, 5.8 \mathrm{eV}$ and $3.2 \mathrm{eV}$, respectively [25]. But the key point in this work is to evaluate the dielectric quality for $\mathrm{VO}_{2}$ based MIT FET with a certain reference criterion $(\mathrm{BO} \sim 1 \mathrm{eV})$, so the specific band gap values are not so significant herein. An antiferromagnetic setting of $\mathrm{VO}_{2}$ is always kept during geometry and electronic calculations, as in our previous reports [26,27].

The realistic gate dielectric prepared by deposition techniques (e.g., atomic layer deposition or chemical vapor deposition) commonly have an amorphous structure. But the essential requirement for interface modeling is the atomic local bonding rather than long-range crystalline symmetry. The monoclinic $\mathrm{M} 1$ phase $\mathrm{VO}_{2}$ was strained to an orthorhombic structure to match the crystalline insulators. We consider three types of interfaces, namely, a high dielectric constant crystalline insulator (cubic $\mathrm{HfO}_{2}$ and $\mathrm{ZrO}_{2}$ ), a wide band gap insulator (modified $\theta-\mathrm{Al}_{2} \mathrm{O}_{3}$ ) and a narrower band gap crystalline insulator (anatase $\mathrm{TiO}_{2}$ ). $\mathrm{Al}_{2} \mathrm{O}_{3}$ has several symmetric crystalline phases, among which the most stable one is the hexagonal $\alpha-\mathrm{Al}_{2} \mathrm{O}_{3}$ phase (i.e., corundum, or sapphire) [28], but the mass density $\left(\sim 4.0 \mathrm{gm} / \mathrm{cm}^{3}\right)$ and bandgap $(\sim 8.8 \mathrm{eV})$ in $\alpha-\mathrm{Al}_{2} \mathrm{O}_{3}$ is too high compared to the amorphous $\mathrm{Al}_{2} \mathrm{O}_{3}$, and thus not preferable in calculation. In this work, we adopted a modified $\theta$ phase $\mathrm{Al}_{2} \mathrm{O}_{3}$ structure which has a mass density $\left(\sim 3.5 \mathrm{~g} / \mathrm{cm}^{3}\right)$, bandgap $(\sim 6.6 \mathrm{eV})$ and atomic coordination similar to the amorphous structure grown by ALD [28,29]. To build the interface model, the $\theta$-phase $\mathrm{Al}_{2} \mathrm{O}_{3}$ was reshaped to an orthorhombic structure to match $\mathrm{VO}_{2}$. Cubic $\mathrm{HfO}_{2}$ $\left(\mathrm{ZrO}_{2}\right)$ and anatase $\mathrm{TiO}_{2}$ are found to form a reasonable lattice matched interface to the M1 phase of $\mathrm{VO}_{2}$. All the interface supercells are built symmetrically by adopting the two-interface modeling without vacuum and passivation layer [30]. The lattice is allowed to relax only in the z-direction to release the internal stress.
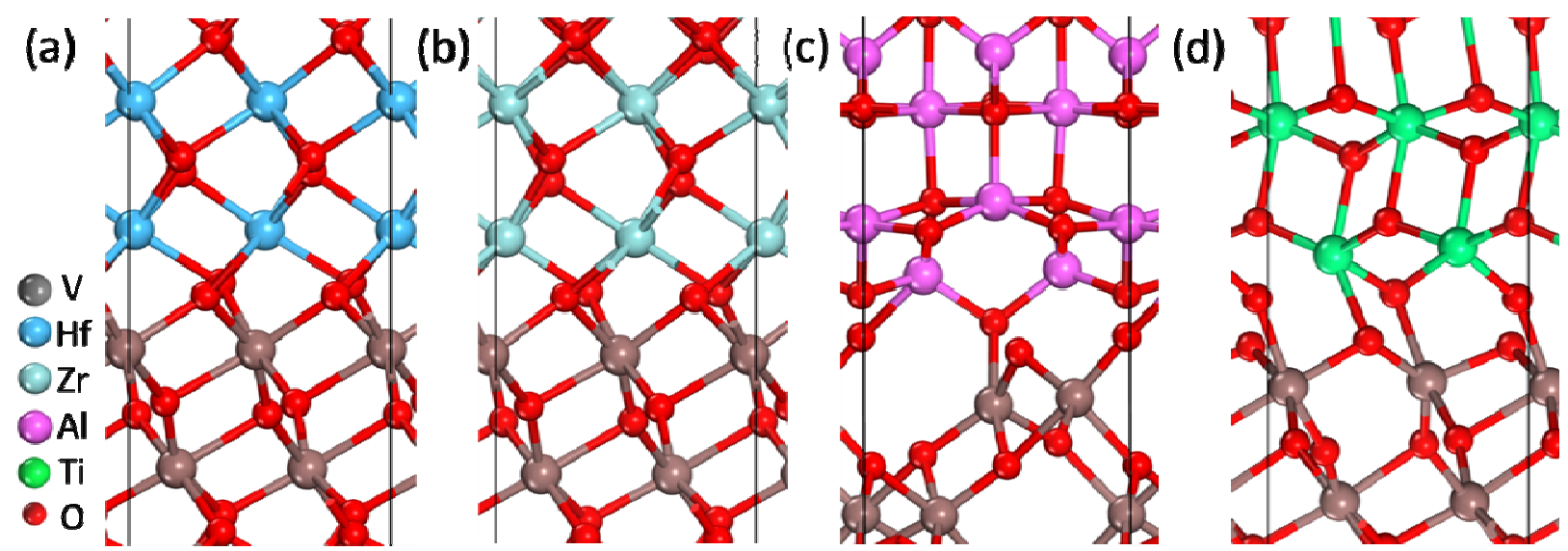

Fig. 1. The atomic structures of final relaxed (a) $\mathrm{HfO}_{2} / \mathrm{VO}_{2}$, (b) $\mathrm{ZrO}_{2} / \mathrm{VO}_{2}$, (c) $\mathrm{Al}_{2} \mathrm{O}_{3} / \mathrm{VO}_{2}$ and (d) $\mathrm{TiO}_{2} / \mathrm{VO}_{2}$ interfaces. The species of atoms are indicated in the insert.

In terms of the theoretical calculation, the accurate band offset values should be obtained from the insulating interface model with no gap states emerging and Fermi level lying at midgap. We 
built the interface models based on the electron counting rule (ECR) to achieve this goal [29,31]. The interface geometry is chosen so that there are no metal-metal bonds and the oxygen sites are bonded to similar numbers of metal sites as in the bulk materials. The interface with metal-O bonding is also more energetically favorable.

\section{Results}

The final relaxed atomic structures of dielectric $/ \mathrm{VO}_{2}$ interfaces are listed in Fig. 1. The final relaxed geometries for $\mathrm{HfO}_{2}$ and $\mathrm{ZrO}_{2}$ interface are almost the same, benefiting from their similar lattice constant and electronic properties, as is shown in Fig. 1(a) and (b). The geometry features the $\mathrm{Hf}-\mathrm{O}(\mathrm{Zr}-\mathrm{O})$ bonds at the interface with no metal-metal bonds. All the $\mathrm{Hf}(\mathrm{Zr})$ atoms are six-fold in the interface supercell, rather than its eight-fold coordination in ideal $\mathrm{HfO}_{2}\left(\mathrm{ZrO}_{2}\right)$ bulk. The different bonding characteristics are owing to the $\sim 5 \%$ lattice mismatch and constrained cell shape in $x$ - and $y$-directions. All the interfacial $\mathrm{O}$ atoms are three-fold, like that in the bulk $\mathrm{VO}_{2} . \mathrm{For} \mathrm{Al}_{2} \mathrm{O}_{3}$ interface in Fig. 1(c), the atomic structure in $\mathrm{Al}_{2} \mathrm{O}_{3}$ bulk side is different from that in the ideal $\theta$ phase $\mathrm{Al}_{2} \mathrm{O}_{3}$, but the local bonding and atomic coordination remain the same. At the interfacial region, the $\mathrm{Al}$ (and $\mathrm{O}$ ) atoms are either five-fold or four-fold (three-fold or two-fold for $\mathrm{O}$ atoms), under-coordinated comparing to the bulk case. The dangling bonds on interfacial $\mathrm{Al}$ and $\mathrm{O}$ atoms will compensate with each other to achieve a closed-shell structure with no gap states. Bonding coordination in $\mathrm{TiO}_{2} / \mathrm{VO}_{2}$ interfacial region is also under-coordinated, similar to that in $\mathrm{Al}_{2} \mathrm{O}_{3}$ one (Fig. 1(d)).

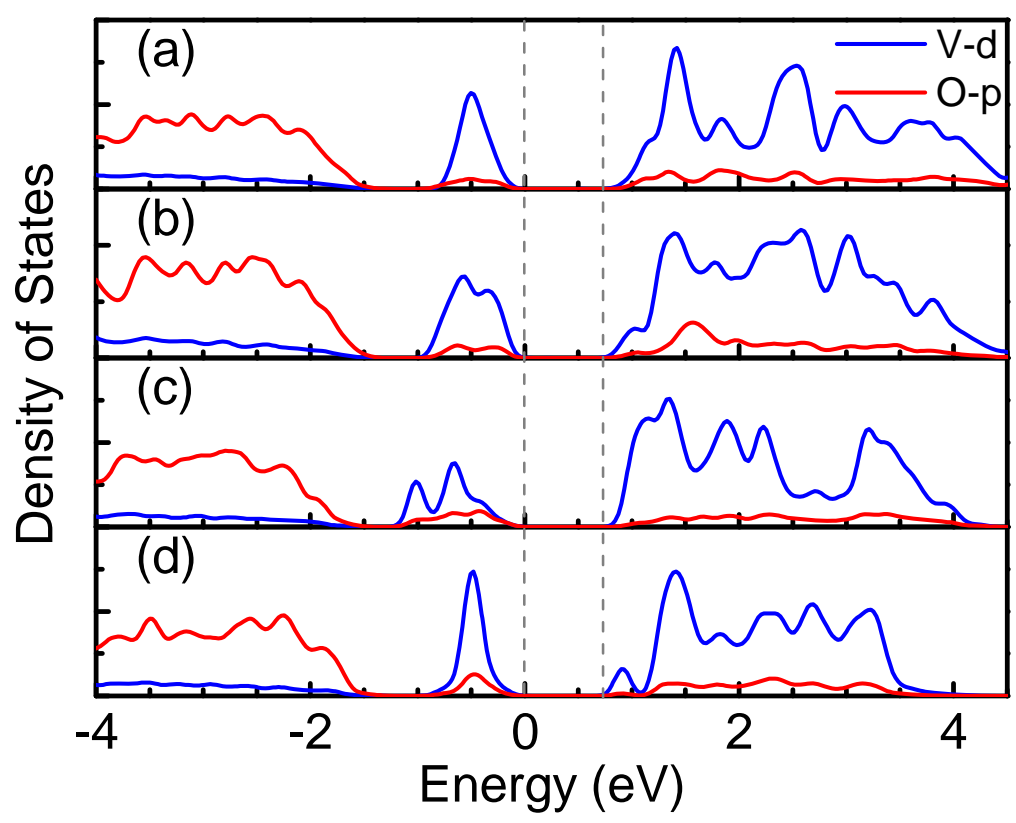

Fig. 2. Partial DOS of the $\mathrm{V}-3 d$ and $\mathrm{O}-2 p$ orbitals of $\mathrm{VO}_{2}$ bulk atoms that lie far away from the interface region in the (a) $\mathrm{HfO}_{2} / \mathrm{VO}_{2}$, (b) $\mathrm{ZrO}_{2} / \mathrm{VO}_{2}$, (c) $\mathrm{Al}_{2} \mathrm{O}_{3} / \mathrm{VO}_{2}$ and (d) $\mathrm{TiO}_{2} / \mathrm{VO}_{2}$ interfaces. $\mathrm{VBM}$ of $\mathrm{VO}_{2}$ bulk is referred to $0 \mathrm{eV}$. The band edges are marked by dashed lines.

The PDOS of the V-3d and O-2p orbitals of $\mathrm{VO}_{2}$ bulk atoms that lie far away from the interface region is shown in Fig. 2. The separation between the highest occupied V- $a_{1 \mathrm{~g}}$ state and O-p state can be observed, which agrees with previous theoretical report $[19,20]$. The bonding $d_{\|}$band shows a sharp peak centering about $0.5 \mathrm{eV}$ below the VBM with a band width of about $1 \mathrm{eV}$, in good agreement with the photoemission experiments [32,33].

The partial density of states (PDOS) of the bulk region $\mathrm{VO}_{2}$ and dielectric atoms that are farthest away from the contact region in the interface supercell are shown in Fig. 3. The valence band maximum (VBM) of $\mathrm{VO}_{2}$ PDOS is referred to $0 \mathrm{eV}$ for convenience. It is seen that the four dielectric $/ \mathrm{VO}_{2}$ models all feature an insulating interface with no gap states occurring in the bandgap, benefiting from the perfect satisfaction of the electron-counting rule. The Fermi-level lies in the 
mid-band gap (not shown). Clearly, the band edge positions of the dielectric side all straddle the band edges of $\mathrm{VO}_{2}$, i.e., the type-I band alignment.
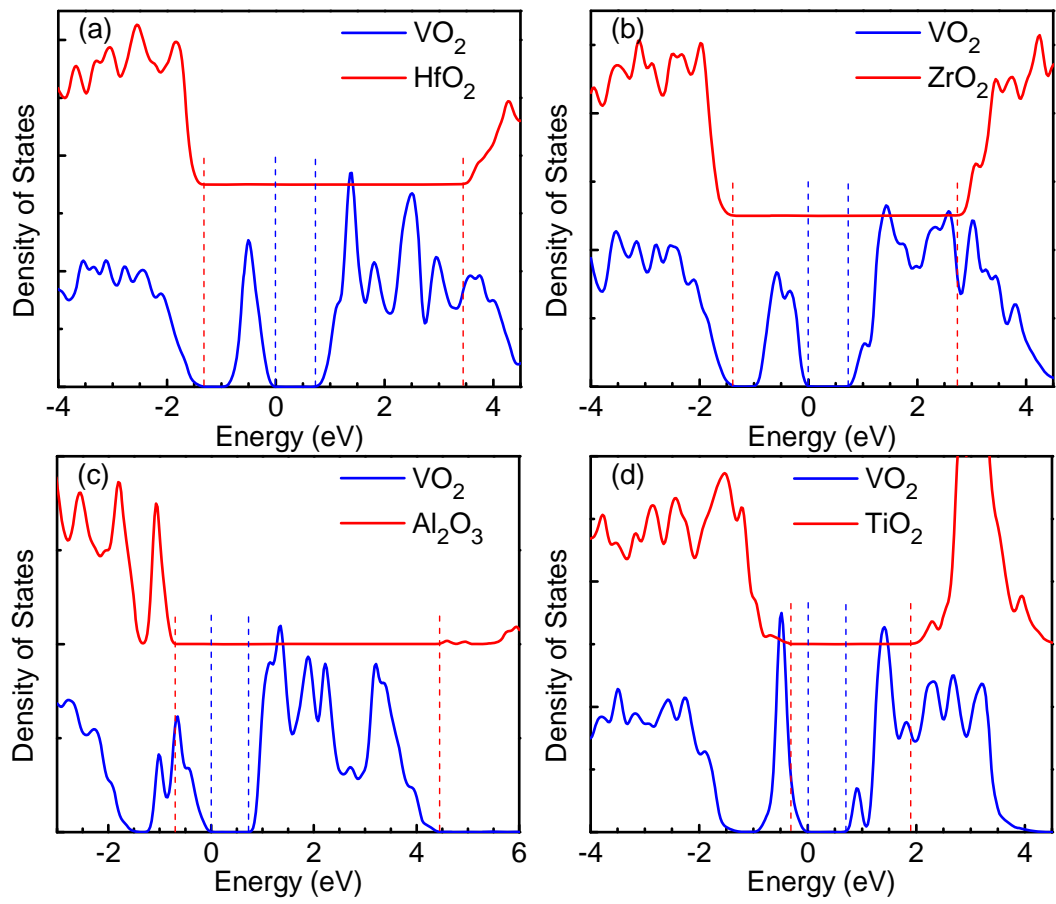

Fig. 3. Partial DOS of the bulk atoms of $\mathrm{VO}_{2}$ and dielectrics that lie far away from the interface region in the (a) $\mathrm{HfO}_{2} / \mathrm{VO}_{2}$, (b) $\mathrm{ZrO}_{2} / \mathrm{VO}_{2}$, (c) $\mathrm{Al}_{2} \mathrm{O}_{3} / \mathrm{VO}_{2}$ and (d) $\mathrm{TiO}_{2} / \mathrm{VO}_{2}$ interfaces, used to obtain the band offsets. $\mathrm{VBM}$ of $\mathrm{VO}_{2}$ bulk is referred to $0 \mathrm{eV}$. Band edges are marked by dashed lines.

Suitable band edge discontinuities (i.e., band offsets) is a crucial requirement for the efficient operation of field-effect transistors [12,25]. The PDOS scheme can be used to roughly determine the band edge line-up in the interface supercell model [30,34]. Using this method, the energy difference between their valence band maxima (i.e., VBO) is roughly observed to be $1.3 \mathrm{eV}, 1.4 \mathrm{eV}$, $0.7 \mathrm{eV}$ and $0.4 \mathrm{eV}$ for $\mathrm{HfO}_{2} / \mathrm{VO}_{2}, \mathrm{ZrO}_{2} / \mathrm{VO}_{2}, \mathrm{Al}_{2} \mathrm{O}_{3} / \mathrm{VO}_{2}$ and $\mathrm{TiO}_{2} / \mathrm{VO}_{2}$ interfaces in Fig. 3, respectively. The counterpart conduction band offsets can be derived as the energy difference between the calculated the band gaps values $\left(4.7 \mathrm{eV}\right.$ for $\mathrm{HfO}_{2}, 4.1 \mathrm{eV}$ for $\mathrm{ZrO}_{2}, 5.2 \mathrm{eV}$ for $\mathrm{Al}_{2} \mathrm{O}_{3}$ and $2.3 \mathrm{eV} \mathrm{TiO}_{2}$ ) and VBOs. But the accuracy of PDOS scheme may be impacted by the band edge distortion induced by the surface reconstruction. In this work, we pay attention to the more accurate method using the core-level state to determine the band edge line-up [35]. The mechanism is described elsewhere [29].

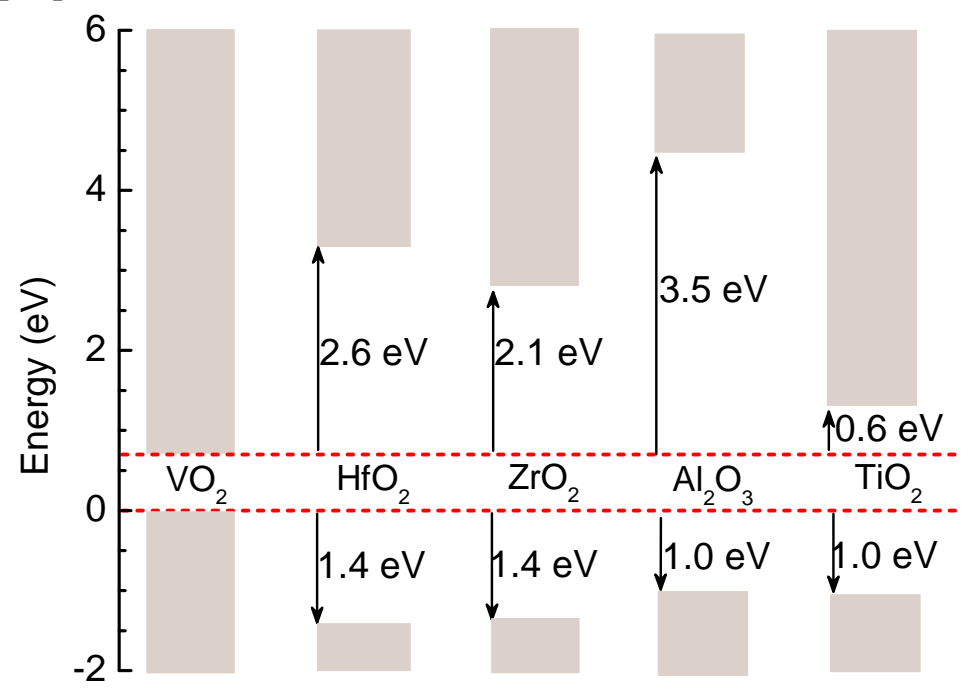


Fig. 4. Schematic band alignment diagram of $\mathrm{VO}_{2}$ with different dielectrics. The VBOs are determined using the core-level alignment scheme, which provides more accuracy. The CBOs are defined as the energy difference between the calculated bandgap and VBO values.

The schematic band alignment diagram of dielectric $/ \mathrm{VO}_{2}$ interface based on the core-level alignment method is shown in Fig. 4. Note that to make sure the band gap of $\mathrm{VO}_{2}$ corresponds to experimental data $(\sim 0.7 \mathrm{eV})$, a smaller $\mathrm{HF}$ fraction of $15 \%$ is used. As a result, the calculated band gaps are $4.7 \mathrm{eV}, 4.1 \mathrm{eV}, 5.2 \mathrm{eV}$, and $2.3 \mathrm{eV}$ for $\mathrm{HfO}_{2}, \mathrm{ZrO}_{2}, \mathrm{Al}_{2} \mathrm{O}_{3}$, and $\mathrm{TiO}_{2}$, respectively, all smaller than the experimental counterpart $\left(6.0 \mathrm{eV}\right.$ for $\mathrm{HfO}_{2}, 5.8 \mathrm{eV}$ for $\mathrm{ZrO}_{2}, 6.5 \mathrm{eV}$ for $\mathrm{Al}_{2} \mathrm{O}_{3}$, and $3.2 \mathrm{eV}$ for $\mathrm{TiO}_{2}$, respectively). Since this work focuses on the dielectric quality in terms of band offset with a certain reference criterion $(\mathrm{BO} \sim 1 \mathrm{eV})$, the specific band gap values are not so significant herein. From Fig. 4, we can see, the VBOs for these four gate dielectrics are all larger than (or equal to) $1 \mathrm{eV}$. Here the similarity of VBOs in $\mathrm{HfO}_{2} / \mathrm{GaN}$ and $\mathrm{ZrO}_{2} / \mathrm{GaN}$ is owing to the VBMs of the two oxides are both nonbonding O-p states and so they lie at similar energy, so that the oxide bandgap narrows by lowering CBM edge with little shift of the VBM. This agrees with our previous work on trivalent oxide and divalent oxide $[29,36]$. The interface dipole, induced by the charge transfer across the interface, has already been explicitly included in the VBO values obtained by our supercell calculations. Meanwhile, since the oxygen ion density is always the same when constructing our models, the VBO is not impacted by the oxygen ion density [37], as is verified by us previously $[38,39]$.

Taking advantage of the calculated oxide bandgap, the corresponding CBO is derived to be 2.6 $\mathrm{eV}, 2.1 \mathrm{eV}, 3.5 \mathrm{eV}$ and $0.6 \mathrm{eV}$, respectively. Obviously, the high dielectric constant insulators all have sufficiently large BOs to confine electrons in $\mathrm{VO}_{2}$ based MIT FET effectively. But the CBO of $\mathrm{TiO}_{2}$ interface is too small, only $0.6 \mathrm{eV}$. As mentioned, it is because of the severe underestimation of $\mathrm{TiO}_{2}$ bandgaps. More effective method on $\mathrm{TiO}_{2} / \mathrm{VO}_{2}$ interface such as parameter-independent hybrid functional (e.g., screened exchange (sX) functional) or LDA+U (different U values on V-d and Ti-d orbitals) will be further considered. If using the experimental bandgap instead of the calculated one here, the newly derived $\mathrm{CBO}$ is $\sim 1.5 \mathrm{eV}$ for $\mathrm{TiO}_{2} / \mathrm{VO}_{2}$ interface, satisfying the $1 \mathrm{eV}$ criterion for confining electrons at semiconductor side $[12,25]$.

\section{Conclusions}

In conclusion, hybrid density functional calculation was conducted to study the dielectric quality for $\mathrm{VO}_{2}$ based devices. High dielectric constant $\mathrm{HfO}_{2}$ and $\mathrm{ZrO}_{2}$, wide band gap oxide $\mathrm{Al}_{2} \mathrm{O}_{3}$, and narrower band gap $\mathrm{TiO}_{2}$ are considered. The insulating interface supercells show proper band alignment for these oxide/ $\mathrm{VO}_{2}$ interfaces. For $\mathrm{HfO}_{2}, \mathrm{ZrO}_{2}$ and $\mathrm{Al}_{2} \mathrm{O}_{3}$, the band offset are well enough. But more accurate line-up analysis between $\mathrm{TiO}_{2}$ and $\mathrm{VO}_{2}$ is still needed.

We thank the financial support from the European Union's Horizon 2020 research and innovation program PHASECHANGE SWITCH (Grant No. 737109). We also acknowledge the support from Supercomputing Wales under the project SCW1070.

\section{References}

[1] F.J. Morin, Phys. Rev. Lett 3 (1959) 34.

[2] J.B. Goodenough, J. Solid State Chem. 3 (1971) 490.

[3] K. Liu, S. Lee, S. Yang, O. Delaire, J. Wu, Mater. Today 21 (2018) 875.

[4] Y. Gao, H. Luo, Z. Zhang, L. Kang, Z. Chen, J. Du, M. Kanehira, C. Cao, Nano Energy 1 (2012) 221.

[5] E.A. Casu, N. Oliva, M. Cavalieri, A.A. Muller, A. Fumarola, W.A. Vitale, A. Krammer, A. Schuler, M. Fernandez-Bolanos, A.M. Ionescu, IEEE J. Electron Devices Soc. 6 (2018) 965.

[6] N. Shukla, A.V. Thathachary, A. Agrawal, H. Paik, A. Aziz, D.G. Schlom, S.K. Gupta, R. Engel-Herbert, S. Datta, Nat. Commun. 6 (2015) 7812. 
[7] C. Ko, S. Ramanathan, Appl. Phys. Lett. 93 (2008) 252101.

[8] D. Ruzmetov, G. Gopalakrishnan, C. Ko, V. Narayanamurti, S. Ramanathan, J. Appl. Phys. 107 (2010) 114516.

[9] J. Son, S. Rajan, S. Stemmer, S. James Allen, J. Appl. Phys. 110 (2011) 084503.

[10] D.M. Newns, J.A. Misewich, C.C. Tsuei, A. Gupta, B.A. Scott, A. Schrott, Appl. Phys. Lett. 73 (1998) 780.

[11] Z. Yang, C. Ko, S. Ramanathan, Annu. Rev. Mater. Res. 41 (2011) 337.

[12] J. Robertson, J. Vac. Sci. Technol. B 18 (2000) 1785.

[13] F. Cerbu, H.S. Chou, I.P. Radu, K. Martens, A.P. Peter, V.V. Afanas'Ev, A. Stesmans, Phys. Status Solidi C 12 (2015) 238.

[14] H. Zhou, M.F. Chisholm, T. Yang, S.J. Pennycook, J. Narayan, J. Appl. Phys. 110 (2011) 073515.

[15] T. Yajima, T. Nishimura, A. Toriumi, Nat. Commun. 6 (2015) 10104.

[16] C. Zhu, S.L. Caudle, J. Yang, D.J. Smith, R.J. Nemanich, J. Vac. Sci. Technol. A 32 (2014) 011203.

[17] C. Zhu, M. Kaur, F. Tang, X. Liu, D.J. Smith, R.J. Nemanich, J. Appl. Phys. 112 (2012) 084105.

[18] L.L. Fan, S. Chen, Z.L. Luo, Q.H. Liu, Y.F. Wu, L. Song, D.X. Ji, P. Wang, W.S. Chu, C. Gao, C.W. Zou, Z.Y. Wu, Nano Lett. 14 (2014) 4036.

[19] V. Eyert, Phys. Rev. Lett. 107 (2011) 016401.

[20] F. Iori, M. Gatti, A. Rubio, Phys. Rev. B 85 (2012) 115129.

[21] S.J. Clark, M.D. Segall, C.J. Pickard, P.J. Hasnip, M.J. Probert, K. Refson, M.C. Payne, Z. Kristallogr. 220 (2005) 567.

[22] J.P. Perdew, K. Burke, M. Ernzerhof, Phys. Rev. Lett. 77 (1996) 3865.

[23] J. Heyd, G.E. Scuseria, M. Ernzerhof, J. Chem. Phys. 124 (2006) 219906.

[24] S. Xu, X. Shen, K.A. Hallman, R.F. Haglund, S.T. Pantelides, Phys. Rev. B 95 (2017) 125105.

[25] J. Robertson, J. Vac. Sci. Technol. A 31 (2013) 050821.

[26] Y. Guo, J. Robertson, Microelectron. Eng. 109 (2013) 278.

[27] Y. Guo, S.J. Clark, J. Robertson, J. Chem. Phys. 140 (2014) 054702.

[28] D. Liu, S.J. Clark, J. Robertson, Appl. Phys. Lett. 96 (2010) 032905.

[29] Z. Zhang, Y. Guo, J. Robertson, Appl. Phys. Lett. 114 (2019) 161601.

[30] P.W. Peacock, J. Robertson, Phys. Rev. Lett. 92 (2004) 057601.

[31] M.D. Pashley, Phys. Rev. B 40 (1989) 10481.

[32] K. Okazaki, H. Wadati, A. Fujimori, M. Onoda, Y. Muraoka, Z. Hiroi, Phys. Rev. B 69 (2004) 165104.

[33] S. Shin, S. Suga, M. Taniguchi, M. Fujisawa, H. Kanzaki, A. Fujimori, H. Daimon, Y. Ueda, K. Kosuge, S. Kachi, Phys. Rev. B 44 (1990) 4993.

[34] Z. Zhang, R. Cao, C. Wang, H. Li, H. Dong, W. Wang, F. Lu, Y. Cheng, X. Xie, H. Liu, K. Cho, R. Wallace, W. Wang, ACS Appl. Mater. Interfaces 7 (2015) 5141.

[35] E.A. Kraut, R.W. Grant, J.R. Waldrop, S.P. Kowalczyk, Phys. Rev. Lett. 44 (1980) 1620.

[36] L. Lin, K. Xiong, J. Robertson, Appl. Phys. Lett. 97 (2010) 242902.

[37] K. Kita, A. Toriumi, Appl. Phys. Lett. 94, (2009) 132902.

[38] L. Lin, J. Robertson, Appl. Phys. Lett. 95, (2009) 012906

[39] L. Lin, J. Robertson, J. Appl. Phys. 109, (2011) 094502. 